\title{
Quantum identification system
}

\author{
Miloslav Dušek ${ }^{1}$, Ondřej Haderka ${ }^{2,1}$, Martin Hendrych ${ }^{2,1}$, Robert Myška $^{2,1}$ \\ ${ }^{1}$ Department of Optics, Palacký University, 17. listopadu 50, 772 00 Olomouc, Czech Republic \\ ${ }^{2}$ Joint Laboratory of Optics of Palacky University and the Physical Institute of the Czech Academy of Sciences, \\ 17. listopadu 50, 772 00 Olomouc, Czech Republic
}

\begin{abstract}
A secure quantum identification system combining a classical identification procedure and quantum key distribution is proposed. Each identification sequence is always used just once and new sequences are "refuelled" from a shared provably secret key transferred through the quantum channel. Two identification protocols are devised. The first protocol can be applied when legitimate users have an unjammable public channel at their disposal. The deception probability is derived for the case of a noisy quantum channel. The second protocol employs unconditionally secure authentication of information sent over the public channel, and thus it can be applied even in the case when an adversary is allowed to modify public communications. An experimental realization of a quantum identification system is described.

PACS 03.67D
\end{abstract}

\section{INTRODUCTION}

Electronic communications have become one of the main pillars of the modern society. Their utilization places new demands on the establishment of security of transmitted data. In everyday life there are many situations when it is necessary to conceal the contents of information conveyed over insecure communications lines, such as when databases containing confidential data on citizens are to be distributed among authorities, when financial transactions are performed between banks (or for electronic shopping over the Internet), or when we want to withdraw money from automated teller machines, and, of course, for military and diplomatic purposes.

In all these instances, cryptography proves very helpful. One of the basic cryptographic tasks is to certify the identities of the legitimate users of a communications line (traditionally called Alice and Bob) so that no third party monitoring their identification can impersonate either of them. Moreover, the system must be designed in such a way that after a successful mutual identification even Bob cannot later on pretend to someone else to be Alice and vice versa.

Existing identification systems are merely computationally secure, i.e., they rely on limited advancement of computer power, technologies, and mathematical algorithms in the foreseeable future. The construction of a quantum computer can seriously menace the security of classical identification systems. A quantum identification system was first proposed by C. Crépeau and L. Salvail in [1]. Their identification protocol is based on quantum oblivious transfer [2,3]. Alice and Bob mutually check their knowledge of a common secret string without disclosing it. However, quantum oblivious transfer has been proved insecure against the so-called collective attacks by D. Mayers [4, 5], and H.-K. Lo and H.F. Chau [6]. Although to perform collective attacks is not possible with current technology, recent developments suggest that it might be possible in the near future.

In the protocols proposed here, Alice and Bob check their common secret string in a classical way. To prevent from a later misuse, each identification sequence is used only once and the distribution of a new common secret string is achieved by means of quantum key distribution (QKD). QKD, based on the BB84 protocol [7], has recently been proved secure against any collective attack allowed by quantum mechanics [8.99, and thus it offers unconditional protection even against eavesdroppers possessing unlimited computational and technological power. QKD is capable to provide two users with a random shared secret string, whose secrecy is guaranteed by the fundamental laws of quantum mechanics. Many papers have already been devoted to quantum cryptography. Let us mention only a few of them [7, 10, 11, 12, 13, 14, 15] and the survey [16]. A large bibliography may also be found in [17].

In this paper, two protocols for quantum mutual identification are presented. The first is designed for the case of an unjammable public channel. Since this requirement might appear too strong in practice, we also present a second protocol that utilizes unconditionally secure authentication of messages sent over the public channel. Both protocols have been implemented in a laboratory setup over a distance of $0.5 \mathrm{~km}$.

\section{IDENTIFICATION WITH UNJAMMABLE OPEN CHANNEL}

On the assumption that the open channel used for communication during the quantum key distribution cannot be modified, a simple identification protocol can be implemented. The proposed identification protocol does not rely on quantum bit commitment or oblivious transfer, 
but it is based on a simple classical identification method using each time a new identification sequence (i.e., the sequence is changed after each identification act, either successful or unsuccessful). This method is secure in the following sense: a sufficient length of an identification sequence (IS) exists such that the probability of a success of an unauthorized user is smaller than an arbitrary small positive number. Since each IS may be used only once, the users need to regularly refuel their pools of sequences. Here we are coming to the "quantum part" of the protocol. The well known quantum key distribution procedure (QKD) [7] is well suitable to accomplish this task. Of course, a certain amount of secret information must be shared at the beginning. But later, the used ISs are replaced by new ones transmitted by means of QKD. A limited number of ISs could be stored, e.g., on a chip card and encrypted using a "personal identification number" (PIN). Owing to discarding each used IS, the probability of a success of an unauthorized user of the "lost" card depends on the number of stored ISs and on the length of the PIN (varying these parameters, this probability could be made arbitrarily small).

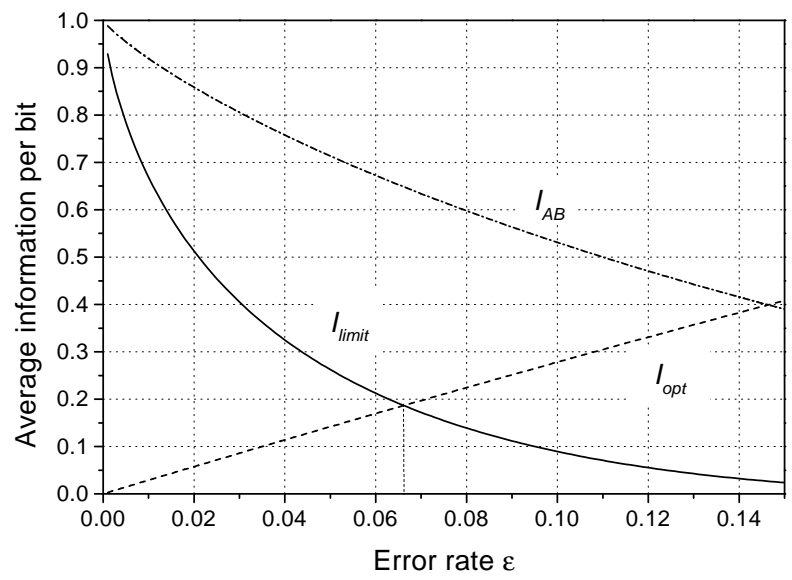

FIG. 1. Information as a function of error rate: $I_{A B}-$ information shared by Alice and Bob, $I_{o p t}-$ Eve's information gained using optimum eavesdropping strategy according to [21], $I_{\text {limit }}$ - below this limit of Eve's information on the key, the deception probability in Protocol I can be made arbitrarily small by prolonging ISs. The intersection of $I_{\text {limit }}$ and $I_{o p t}$ shows the estimate of the upper bound of the error rate for the identification application following Protocol I.

Let us note that there is no need to perform error correction and privacy amplification [10] after QKD. The correspondence between two compared ISs need not then be perfect, the errors being caused either by the imperfections of the device or by eavesdropping. If the legitimate parties tolerate a certain small number of errors, then it is also necessary to suppose that an eavesdropper (Eve) could capture some information on new "refuelled" ISs by measurements on the quantum channel. The authorized users are able to estimate the amount of this informa- tion [21]. Nevertheless, if the ISs are long enough, this tap information is not sufficient for Eve to succeed in the identification procedure, at least if she can perform only separate and independent measurements on transmitted qubits (for the so-called coherent attack the situation is more complex). Strictly speaking, for error rates below a certain level, the deception probability can be made arbitrarily small by prolonging ISs. For details see Appendix A.

As an example, assume an error rate $\varepsilon=0.01$ (it can be seen in Fig. 1 that this error rate lies below the upper bound value $\varepsilon_{\mathrm{ub}}$ (Eq. A4)). For this error rate, the average probability that Eve correctly guesses a bit, if she applies an optimum strategy, is approximately $\bar{p} \doteq 0.6$ (this is computed from $I_{o p t}-$ see Fig. 1 - using Eq. (65) in [21] and the definition of information). Then it follows from Eq. A2 that for ISs of length $N \geq 50$ bits, the deception probability $P(N, \varepsilon)<10^{-10}$.

The protocol consists of a three-pass exchange of ISs and it can be realized as follows. Note that Alice and Bob must initially share several triads of ISs.

$$
\text { Protocol I (unjammable open channel) }
$$

- Alice and Bob say each other their ordinal numbers of IS triads in the stack - a pointer to the first Alice's (Bob's) unused sequence - and choose the higher one if they differ.

- $\quad$ - Alice sends the first IS of the triad to Bob.

- Bob checks whether it agrees with his copy. If not, Bob aborts communication and shifts his pointer to the next triad. Otherwise, he sends the second IS of the triad to Alice.

- Alice compares whether her and Bob's second ISs agree. If not, she aborts communication and shifts her pointer. Otherwise, she sends the third IS to Bob. If Bob finds it correct, the identification is successfully finished.

- To replace the used ISs, Alice and Bob "refuel" new ISs by means of QKD and set the pointers to their initial positions.

Three passes are necessary for the following reason: An eavesdropper (Eve) can pretend to be Bob and get the first IS from Alice. Of course, Alice recognizes that Eve is not Bob because Eve cannot send the correct second IS. So Alice aborts connection and discards this triad (i.e., shifts the pointer to the next one). However, later on Eve could turn to Bob and impersonate Alice. She knows the first IS! Bob can recognize a dishonest Eve just only because she does not know the third IS.

Another possibility would be to have only one IS and to send alternately one bit from Alice to Bob and one from Bob to Alice. The communication is aborted when an admissible number of errors is exceeded. However, the derivation of deception probability is more complicated in this case. 


\section{IDENTIFICATION WITH AUTHENTICATED PUBLIC DISCUSSION}

In practice, the "auxiliary" information transmitted through the open channel during QKD could be modified, as it is difficult to create a physically unjammable classical channel. Therefore authentication of the messages sent over the open channel is necessary. This procedure requires additional "key" material to be stored and transmitted in a similar way as ISs (again, each "key" may be used just once). This authentication, however, can be utilized for the identification itself. A three-pass authenticated public discussion, performed during QKD, can function as the three-pass exchange of ISs described in the preceeding section.

However, there are several problems. First, it would be more difficult to estimate Eve's chances to succeed in the identification, if a certain number of errors were allowed in the quantum distributed key, because the authentication tag depends not only on the "key" (or IS) but also on the message itself. So, it is necessary (or, at least, simpler) to execute error correction and privacy amplification.

The second problem is more subtle. For quantum cryptography to provide unconditional security, the procedure used for authentication of public discussion must also be unconditionally secure, not only computationally. Such authentication algorithms exist [18. These algorithms are based on the so-called orthogonal arrays 19. It can be shown, however, that the length (in bits, e.g.) of an "authentication key" must always be greater than the length of the authenticated message. If $m$ is the number of all possible messages, $\kappa$ the number of keys, and $n$ the number of all possible authentication tags, it can be proved using methods of orthogonal arrays theory that

$$
\kappa \geq m(n-1)+1
$$

Now it is straightforward to show that

$$
\kappa>m \text {, if } n \geq 2 \text {. }
$$

An example of an authentication protocol is given in Appendix B.

This fact represents a difficulty for QKD. The length in bits of the messages communicated over the public channel is always greater than the length of transmitted "quantum" key. For each qubit at least one bit of information about the basis chosen by Alice and one bit about the basis chosen by Bob must be interchanged. Only about one half of all successfully received qubits can be used as a key (requirement of coincidence of bases). Besides, part of the key has to be sacrificed and compared by Alice and Bob in order to detect potential eavesdropping, which is also done through the open channel. So there is not enough "quantum" key material to replace the used bits for the next authentication even in the case one does not intend to use the transmitted "quantum" key (or its part) for other purposes.
The way out of this impasse is to realize that it is not necessary to authenticate all parts of the public discussion done during QKD.

The most important and characteristic property of quantum cryptography is that any attempt at eavesdropping inevitably increases the number of errors in the transmitted key. Thus it is necessary to prevent Eve from modifying in any way the part of public discussion connected with the error-rate estimation. Therefore, messages containing the sacrificed part of the "quantum" key (including corresponding bases and positions of sacrificed bits) have to be authenticated. Any modification of the rest of public communication could impair QKD, but would not jeopardize the security of the system. This check on error rate should be performed as the first step of the public discussion, even before the establishment of the sifted key by comparison of bases! Otherwise a malicious Eve could manipulate the non-authenticated public transmission for her benefit. She could, e.g., exchange separate sifted keys with Alice and Bob and then choose only those bits where the choice of bases coincides for all three of them, thus obtaining full knowledge of the key without increasing the error rate (at the cost of decreasing the transmission rate).

An important question is the length of the sacrificed subset that serves the error-rate estimation. Alice and Bob agree on a maximum tolerable error rate $\varepsilon_{\max }$, whose value must be lower than the theoretical limit for a safe noisy quantum channel. Several such limits have been derived in the literature for different kinds of Eve's attacks [21.22], nevertheless the ultimate value for the most general attack is not known at present. In Appendix C we give a derivation of the length of the subset and the limiting error-rate estimate $\varepsilon_{\lim }$ Alice and Bob can accept so that the probability that the actual error rate is higher than $\varepsilon_{\max }$, is lower than a prescribed "safety parameter" $\delta$.

Provided that Alice and Bob initially share a pool of secret information, the identification procedure consists of the following steps:

Protocol II (authenticated open channel)

- Alice and Bob first perform transmission over the quantum channel according to the BB84 protocol, i.e., Alice randomly alternates two bases and two bit values, while Bob records detections in randomly chosen bases (raw quantum transmission).

- Alice and Bob say each other their addresses in the pool of shared secret information - a pointer to the first Alice's (Bob's) unused bit - and choose the higher one if they differ. Then follows a three-pass authenticated public discussion that serves the estimation of the error rate and mutual identification:

- Bob sends to Alice an authenticated message containing the positions of bits randomly selected for error-rate estimation. 
- Alice checks authentication and aborts communication if it fails. Otherwise she sends back to Bob an authenticated message containing the bases and bit values of the selected qubits.

- Bob checks authentication and aborts communication if it fails. Next he compares bases of the selected subset and retains only those qubits where his and Alice's bases coincide. At last, he estimates error rate. He sends to Alice an authenticated message to inform her that identification was successful and to convey the value of the error-rate estimate. Alice checks authentication and aborts communication if it fails.

- If the error-rate estimate is lower than a maximum tolerable error rate $\varepsilon_{\text {lim }}$, Alice and Bob compare bases of the rest of their raw data and arrive at their sifted keys. Otherwise they suspect Eve of listening in and cannot safely use the just accomplished quantum transmission to establish new shared secret sequences.

- Then they perform error correction and privacy amplification procedures and arrive at an error-free distilled key. The level of privacy amplification is based on $\varepsilon_{\max }$.

- Alice and Bob refuel their shared secret information.

The used authentication sequences are always thrown away. The length of the raw quantum transmission must be selected such that the length of the newly obtained distilled key is greater than the number of bits consumed for authentication/identification purposes. It is convenient if it covers several unsuccessful identification acts. We give concrete figures in Section $\square$.

\section{DESCRIPTION OF THE APPARATUS}

The experimental implementation of our system is based on an interferometric setup (i.e., on phase coding) with time multiplexing. It consists of two unbalanced fibre Mach-Zehnder interferometers (see Fig. 2). The path difference $(2 \mathrm{~m})$ of the arms of each interferometer is larger than the width of the laser pulse (its duration is $4 \mathrm{~ns}$ ). Interference occurs at the outputs of the second interferometer for pulses "taking" long-short or short-long paths. These paths are of the same length and are indistinguishable. Each of these interferometers represents the main part of the "terminals" of both communicating parties. The terminals are interconnected by a $0.5 \mathrm{~km}$ single mode optical fibre acting as a quantum channel, and by a classical channel (local computer network). As a light source, a semiconductor pulsed laser with a repetition rate of $100 \mathrm{kHz}$ operating at $830 \mathrm{~nm}$ is used. Laser pulses are attenuated by a computercontrolled attenuator so that the intensity level at the output of the first interferometer is below 1 photon per pulse on the average. The accuracy of this setting is monitored by detector D3. Polarization properties of light in the interferometers are controlled by polarization controllers PoC. To balance the lengths of the arms, an air gap AG with a remotely controlled gap-width is used. The phase coding is performed by means of two planar electro-optic phase modulators PM (one at each terminal). To achieve high interference visibility, the splitting ratio of the last combiner must approach 50:50 as closely as possible (see 20]). Therefore a variable ratio coupler VRC is employed there. With this setup, it is possible to reach visibilities well above $99.5 \%$. The total losses of the second interferometer do not exceed $4.5 \mathrm{~dB}$.

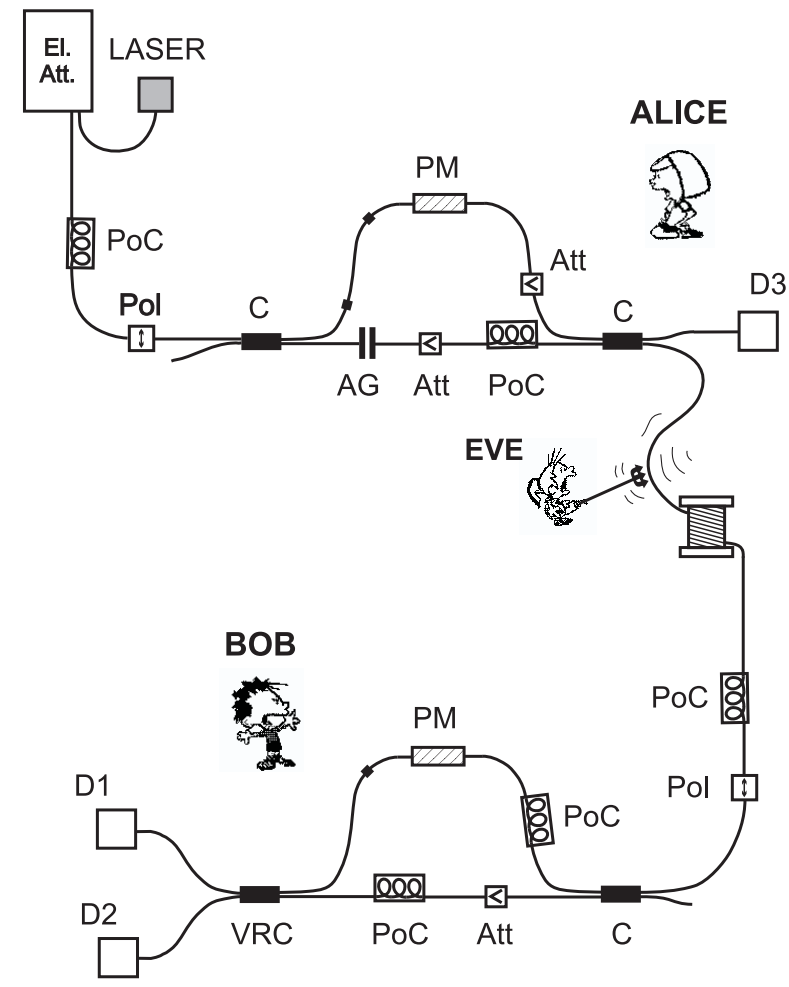

FIG. 2. A scheme of the optical part of the built quantum identification system. El. Att. - electronic attenuator, PoC - polarization controllers, PM - planar electro-optic phase modulators, ATT - attenuators, Pol - polarizers, C - fibre couplers, VRC - variable ratio coupler, AG - air gap.

Detectors D1-D3 are single photon counting modules with Si-avalanche photodiodes. Their output signals are processed by detection electronics based on time-toamplitude converters and single channel analyzers. Both terminals are fully driven by computers. The interferometers are placed in polystyrene thermo-isolating boxes. Together with automatic active stabilization of interference, it enables us to reach low error rates (0.3-0.4\%) with data transmission rates of the order of several kbits 
per second.

\section{PRACTICAL IMPLEMENTATION (PROTOCOL II)}

To estimate the error rate of the just completed quantum transmission, Alice and Bob sacrifice a subset of their raw data and publicly compare bit values. It is important that this is the first step of processing the raw data obtained from the transmission over the quantum channel. The selection of bit positions for the subset must be completely random so that Eve has no a priori information about which bits may appear in the subset.

Let us first focus on the authenticated part of public discussion. We choose the length of the subset $2 s=2000$ bits. If the "safety parameter" $\delta=10^{-10}$ is required, we must reject all raw quantum transmissions for which we obtain error-rate estimate $\varepsilon_{\text {est }} \geq 2.4 \%$ (see Appendix $\mathrm{C}$ for details). If the total of $N$ laser pulses have been used for the raw quantum transmission, we need

- $2 s\left[\log _{2} N\right]+a$ bits to convey and authenticate positions of selected bits,

- $4 s+a$ bits to convey authenticated bases and bit values of the selected bits, and,

- say, $32+a$ bits to convey the final message whether everything is OK or not.

Here $[x]$ denotes the smallest integer larger than $x$, and $a \geq\left[\log _{2}(1 / \delta)\right]$ is the length of the authentication tag (see Appendix B). We use $a=61$. In total this gives the requirement to share at least

$$
b_{\min }=2 s\left(\left[\log _{2} N\right]+2\right)+32+3 a
$$

secret bits initially.

The length of the sifted key we obtain depends on the intensity of laser pulses $\mu$ at the output of Alice's interferometer, on the transmissivity of the communications line $\eta_{\mathrm{TL}}(0.63$ in our device), transmissivity of Bob's interferometer $\eta_{\mathrm{BOB}}(0.35)$, and the quantum efficiency of detectors $\eta_{\mathrm{DET}}(0.55)$. This yields overall transmissivity $\eta=\eta_{\mathrm{TL}} \eta_{\mathrm{BOB}} \eta_{\mathrm{DET}}=0.12$ and we obtain a sifted key of the average length

$$
N_{S}=\frac{1}{2} \eta \mu N
$$

The error correction and privacy amplification procedures we use are basically those used by Bennett et al. [10. We have empirically found that after error correc-

\footnotetext{
*The improved techniques of 23,24, enabling a more rigorous determination of the fraction of key bits to be discarded [25], can also be used, and very recent results of [26] show how to incorporate more sophisticated quantum non-demolition measurement instead of a beamsplitting attack.
}

tion we are left with approximately

$$
N_{C}=\left(1-2.7 \varepsilon^{2 / 3}\right) N_{S}
$$

bits, $\varepsilon$ being the actual error rate. At last, privacy amplification leaves us with

$$
\begin{aligned}
N_{D} & =N_{C}-\frac{\eta \mu^{2}}{8 \eta_{\mathrm{TL}}} N-\frac{2 \varepsilon_{\max } N_{S}}{\ln 2} \\
& -5 \sqrt{N \frac{\eta \mu^{2}}{8 \eta_{\mathrm{TL}}}\left(1-\frac{\eta \mu^{2}}{8 \eta_{\mathrm{TL}}}\right)+\frac{2(\ln 2+1) N_{S} \varepsilon_{\max }}{\ln ^{2} 2}} \\
& +\frac{\ln (\delta \ln 2)}{\ln 2}
\end{aligned}
$$

bits of distilled keyt. The second term on the r.h.s. of Eq. (3) expresses the number of bits Eve could obtain by beamsplitting [10] with the capability of replacement of the lossy communications line by a line of $\eta_{\mathrm{TL}}=1$, while Alice and Bob tolerate a drop in the data rate to one half of the expected value $\$$. The third term contains the number of bits Eve could obtain by a probe interaction attack with the possibility of delayed (after the announcement of the bases but before error correction and privacy amplification) measurements on individual photons 21 fourth term is a 5-standard-deviations safeguard, whose derivation is analogous to that in [10]). The last term is a privacy amplification compression that decreases Eve's information to $\delta$ bits. Collective attacks are not included, as no bound on the information an eavesdropper can get through a collective attack has been derived yet; it has just been proved that such a bound exists 8.9. Preliminary results on coherent eavesdropping also suggest that it does not seem to substantially increase Eve's information 27].

We have optimized this relation to maximize the ratio $N_{D} / N$. For our system $(\eta=0.12, \varepsilon=0.004)$ with the choice $\varepsilon_{\max }=0.07$ and $\left.2 s=2000\right)$, we have found an optimum average intensity $\mu \approx 0.8$ photon per pulse (Fig. 3). This value represents a trade-off between the number of pulses successfully detected by Bob and the reduction of the length of the key caused by privacy amplification, and sensitively depends on the overall losses of the system. The ratio $N_{D} / N$ depends only weakly on $\delta$ so that it is easy to achieve an arbitrary security level.

It is worth noting that for sufficiently low $\mu$, the ratio $b_{\min } / N_{D}$ converges to zero with increasing $N$ so that it is always possible to generate more new shared secret

\footnotetext{
${ }^{\dagger}$ Eq. (3) is valid for $\mu \ll 1$, a general relation is somewhat clumsy and we do not give it here.

${ }^{\ddagger}$ The intensity at Bob’s side also fluctuates for several physical reasons, therefore it is not reasonable to limit the intensity drop caused by eavesdropping in a too restrictive way.

${ }^{\S}$ In fact, this number represents the information Eve could obtain, which may not necessarily be in the form of a set of deterministic bits.
} 
bits than it is consumed for authentication. Therefore authenticated QKD may be regarded as an "expander" of shared secret information, once the ratio $r=N_{D} / b_{\min }$ is greater than 1. For our system, we get $r=1$ for $N=3.1 \times 10^{6}$ laser pulses (see Fig. 3).

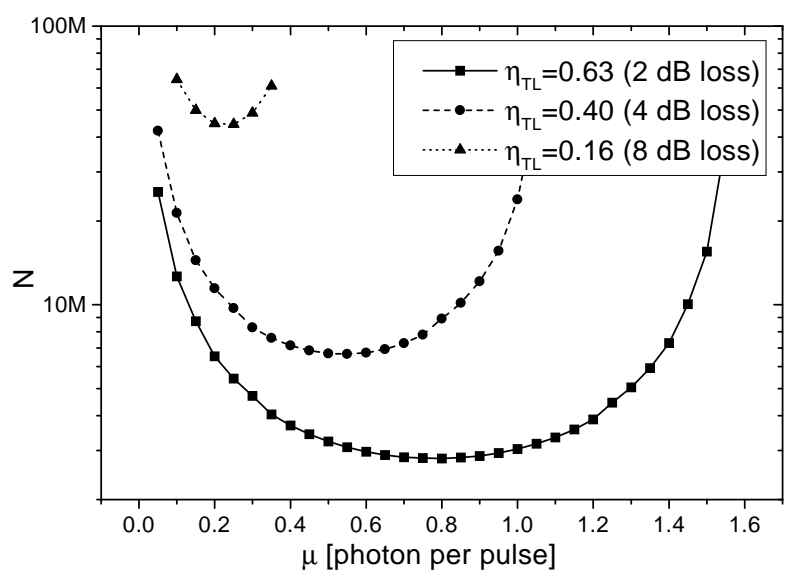

FIG. 3. The dependence of the number of laser pulses $N$ needed to generate as much distilled key (Eq. 3) as it is consumed for authentication during identification (Eq. 1) on the intensity $\mu$ of laser pulses at the output of Alice's interferometer for three different values of the transmissivity of the communications line $\eta_{\mathrm{TL}}$. The higher the losses of the transmission line (or its length), the lower must be the intensity at the output of Alice's interferometer and the greater is the number of laser pulses needed to generate enough distilled key. We can see that the optimum intensity is about 0.8 photon per pulse in our case $\left(\eta_{\mathrm{TL}}=0.63\right)$.

The whole identification procedure starts with raw quantum transmission. In our experimental setup, we generate raw key data at sequences of 320,000 laser pulses. After each sequence, active stabilization of the interferometers is performed to ensure low error rate despite environmental perturbations. This yields an average raw key data rate of cca 5.7 kbits per second. Once about 600,000 photons are successfully detected by Bob (we want $r \geq 2$ ), a three-pass authenticated public discussion is performed according to Protocol II described above. If all three authentications are found correct, Alice and Bob have mutually identified themselves. If, in addition, the computed error-rate estimate falls below the value $\varepsilon_{\text {lim }}$, they are able to refuel new secret key material.

They start doing this by comparing the bases of the rest of the raw key data, thus arriving at approximately 300 kbits of sifted key. As final steps, they perform error correction and privacy amplification procedures. The level of privacy amplification is based on $\varepsilon_{\max }$ and the "security parameter" $\delta$, as follows from Eq. (3). For our usual error rates of $0.3-0.4 \%$, Alice and Bob obtain about 117 kbits of distilled key generated at an average rate of 650 bits per second. This well covers approximately 50 kbits of previously shared secret key material consumed during the authenticated discussion. Let us note that we did not perform any special optimization of data rate, the bottlenecks being here the way we drive the equipment from PCs and the bandwidth of the detection electronics we used. Nevertheless, in our setup the whole identification procedure takes less than 3 minutes (including all auxiliary processes).

\section{CONCLUSIONS}

We have discussed the possibility to utilize the advantages of quantum cryptography for mutual identification. Quantum key distribution can serve as a source to "continuously" supply shared secret key material for classical identification methods, which employ one key just for one identification act. For the case of an unjammable open channel and a noisy quantum channel, a simple identification protocol has been proposed and deception probability has been derived. For a more realistic situation of a jammable open channel, an identification protocol employing authentication of public discussion has been devised. A laboratory prototype of the identification system has been built. It is based on a "one-photon" interferometric method and on the quantum transmission protocol BB84. The main physical parameters are following: distance $0.5 \mathrm{~km}$, wavelength $830 \mathrm{~nm}$, raw data rate about 5 kbits per sec., distilled key generation rate 650 bits per sec., error rate in the range $0.3-0.4 \%$.

\section{ACKNOWLEDGEMENTS}

The authors wish to express their gratitude to Professor Jan Peřina for his help throughout and for encouragement. This research was supported by Czech Ministry of Education (VS 96028), Czech Grant Agency (202/95/0002) and Czech Home Department (19951997007, 19982003012).

\section{APPENDIX A: DECEPTION PROBABILITY}

Let us denote $\varepsilon$ the error rate of the device. Let the length of IS be $N$ and let us tolerate maximally $k=[\varepsilon N]$ errors in the identification procedure $([x]$ denotes the smallest integer greater than $x$ ). If Eve's measurements are independent and if the probability that Eve correctly guesses the $i$-th bit in the sequence is $p_{i}$, then the probability that Eve succeeds in the identification is

$$
P(N, \varepsilon)=\sum_{\ell=0}^{k} \sum_{\left\{i_{1} \ldots i_{\ell}\right\}}\left(\prod_{j=1}^{N} p_{j}\right)\left(\prod_{m=1}^{\ell} \frac{q_{i_{m}}}{p_{i_{m}}}\right) .
$$

Here $q_{i}=\left(1-p_{i}\right)$ and the second sum goes over all $\ell$ tuples of numbers from 1 to $N$ (for $\ell=0$ there is only 
$\left.\prod_{j} p_{j}\right)$. Employing Jensen's inequality [28], one can find that

$$
\prod_{j=1}^{N} p_{j} \leq(\bar{p})^{N}
$$

with

$$
\bar{p}=\frac{1}{N} \sum_{j=1}^{N} p_{j} .
$$

Further, realizing that for $p \geq 1 / 2$ the expression $q / p \leq 1$ is valid and that for $\ell \leq k$ the inequality $\left(\begin{array}{l}N \\ \ell\end{array}\right) \leq\left(\begin{array}{l}k \\ \ell\end{array}\right)\left(\begin{array}{c}N \\ k\end{array}\right)$ holds, one finally obtains the deception probability:

$$
P(N, \varepsilon) \leq(\bar{p})^{N} 2^{k}\left(\begin{array}{l}
N \\
k
\end{array}\right) .
$$

The question is for which $\bar{p}$ and $\varepsilon$ the $\lim _{N \rightarrow \infty} P(N, \varepsilon)=$ 0 [i.e., when $P$ can be made arbitrarily small by increasing $N$ ]. It can be shown that if $0<\lim _{N \rightarrow \infty} \beta(N)<1$ then $\lim _{N \rightarrow \infty}[\beta(N)]^{N}=0$ and if $\lim _{N \rightarrow \infty} \beta(N)>1$ then $\lim _{N \rightarrow \infty}[\beta(N)]^{N}=\infty(\beta(N)$ is an arbitrary function of $N)$. Thus for each $\varepsilon$, a probability $p_{\text {crit }}$

$$
p_{\text {crit }}=\lim _{n \rightarrow \infty} 2^{-k / n}\left(\begin{array}{l}
n \\
k
\end{array}\right)^{-1 / n},
$$

may be defined such that for all $\bar{p}<p_{\text {crit }}$, the limit $\lim _{N \rightarrow \infty} P(N, \varepsilon)=0$. The graph in Fig. 1 shows average information per bit $I_{\text {limit }}=1+p_{\text {crit }} \log _{2}\left(p_{\text {crit }}\right)+$ $\left(1-p_{\text {crit }}\right) \log _{2}\left(1-p_{\text {crit }}\right)$ corresponding to $p_{\text {crit }}$ together with mutual information of Alice and Bob $I_{A B}$ and Eve's information gained by optimal eavesdropping strategy $I_{o p t}=I_{A E}=I_{E B}$ (see 21] Eq. (65)) as a function of error rate $\varepsilon$. The intersection of $I_{\text {limit }}$ and $I_{\text {opt }}$ determines the estimation of the upper bound of the error rate for this identification application:

$$
\varepsilon_{u b} \approx 0.066 \text {. }
$$

A disputative question might be the case of collective (or coherent) attacks when the probabilities of the correct guesses of particular bits need not be independent anymore and then the probability $P(N, \varepsilon)$ may decrease with increasing $N$ more slowly in comparison with the previous case.

\section{APPENDIX B: EXAMPLE OF AUTHENTICATION PROTOCOL}

If probabilities of impersonification are to be the same for all possible pairs (message, authentication tag), then there exists an orthogonal array that serves as a base for an authentication code. In such a case the deception probability, defined as a maximum from the above mentioned probabilities of impersonification, is minimal and is equal to the reciprocal of the number of all possible authentication tags.

There is a class of orthogonal arrays that enables us to construct reasonable authentication codes [18]. If $p$ is prime and $d \geq 2$ is an integer, an authentication code can be created for $\left(p^{d}-1\right) /(p-1)$ messages with $p^{d}$ keys and $p$ authentication tags (the deception probability is $p^{-1}$ ). For a given message and a given authentication key, the authentication tag can be calculated as follows:

1. Convert a given authentication key to the number system of the base $p$ (its maximal length in this system is $d$ ). Let us denote the $i$-th "digit" by $r_{i}$.

2. Construct and order all non-zero "numbers" in the number system of the base $p$ of the maximum length $d$ that have the first non-zero "digit" from the left equal to 1 [there are $\left(p^{d}-1\right) /(p-1)$ such numbers]. A one-to-one mapping exists between all possible messages and all "numbers" (or sequences) from this set. Assign the corresponding "number" (the ordering of the "numbers" is assumed to be fixed) to the message to be authenticated. Let the $i$-th "digit" of that particular "number" be denoted by $c_{i}$.

3. The authentication tag is given by the equation

$$
A(r, c)=\sum_{i=1}^{d} r_{i} c_{i} \bmod p .
$$

As a practical example (used in implementation of Protocol II), we have chosen $p=2^{61}-1$ (it's prime) and $d=739$. Then the deception probability is about $5 \cdot 10^{-19}$. The length of the key is 45079 bits, the length of the message can be up to 45017 bits and the authentication tag consist of 61 bits.

By the way, in case of $p$ of this form it is not necessary to make the conversion of item (1) above. One can just create groups consisting of 61 random bits. What is only necessary is to discard groups containing all 61 ones (the probability of appearance of such a group is deuced small).

\section{APPENDIX C: DERIVATION OF THE LENGTH OF SUBSET FOR ERROR-RATE ESTIMATION}

Let us suppose that we select a subset of length $2 s$. After comparison of bases, $s$ bits will be retained on the average. Provided that the actual error rate is $\varepsilon$, the probability that we find $k$ errors in the subset of length $s$ (i.e., the error-rate estimate is $\varepsilon_{\text {est }}=k / s$ ) is given by

$$
p\left(\varepsilon_{\text {est }} \mid \varepsilon\right)=\left(\begin{array}{l}
s \\
k
\end{array}\right) \varepsilon^{k}(1-\varepsilon)^{s-k} .
$$

Applying Bayes' theorem, the probability that the actual error rate is $\varepsilon$, when the estimate is $\varepsilon_{\text {est }}=k / s$, is given by 


$$
p\left(\varepsilon \mid \varepsilon_{\text {est }}\right)=\frac{\left\{\varepsilon^{\varepsilon_{\text {est }}}(1-\varepsilon)^{1-\varepsilon_{\text {est }}}\right\}^{s}}{\int_{0}^{1}\left\{\varepsilon^{\varepsilon_{\text {est }}}(1-\varepsilon)^{1-\varepsilon_{\text {est }}}\right\}^{s} d \varepsilon} .
$$

Here we assume a uniform distribution of $\varepsilon$. We are now interested in finding a limiting value $\varepsilon_{\text {lim }}$ such that for all $\varepsilon_{\text {est }} \leq \varepsilon_{\text {lim }}$ the probability

$$
P\left(\varepsilon>\varepsilon_{\max }\right)=\int_{\varepsilon_{\max }}^{1} p\left(\varepsilon \mid \varepsilon_{\text {est }}\right) d \varepsilon \leq \delta,
$$

where a small positive number $\delta$ denotes the "security parameter". In Fig. A we plot the solution of the equation

$$
\frac{\int_{\varepsilon_{\max }}^{1}\left\{\varepsilon^{\varepsilon_{\lim }}(1-\varepsilon)^{1-\varepsilon_{\lim }}\right\}^{s} d \varepsilon}{\int_{0}^{1}\left\{\varepsilon^{\varepsilon_{\lim }}(1-\varepsilon)^{1-\varepsilon_{\lim }}\right\}^{s} d \varepsilon}=\delta
$$

with respect to $\varepsilon_{\lim }$ for several values of $\delta$. A maximum acceptable error rate $\varepsilon_{\max }=0.07$ has been chosen, which is well below the lowest security limit derived so far (0.146) 21]. The graph in Fig. 4 should be understood as follows: Once we select a suitable value for the subset length, $s$, and the "security parameter" $\delta$, the corresponding curve suggests a limiting value for the estimated error level, above which the transmitted sequence should be rejected as it cannot be guaranteed to have the actual error rate $\varepsilon \leq \varepsilon_{\max }$ with the required probability $1-\delta$.

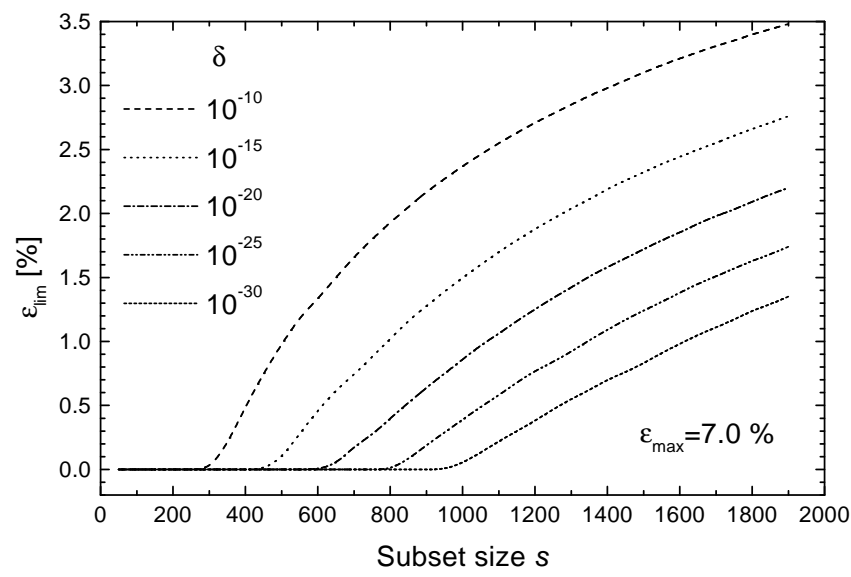

FIG. 4. The dependence of limiting value $\varepsilon_{\text {lim }}$ on a subset size $s$ for different values of the "security parameter" $\delta$, when a maximum error rate of $\varepsilon_{\max }=0.07$ is tolerated (see Eq. C4). A subset of the length $2 s$ is randomly selected from raw quantum data which yields $s$ bits with coincident bases on the average. Quantum transmission is considered insecure (i.e., the probability of the actual error rate $\varepsilon$ being higher than $\varepsilon_{\max }$ is not lower than $\delta$ ), if the error-rate estimate $\varepsilon_{\text {est }}$ obtained from the subset check exceeds the value $\varepsilon_{\text {lim. }}$. In our case we choose $s=1000$ and $\delta=10^{-10}$, and we find $\varepsilon_{\lim } \approx 2.4 \%$.
[1] C. Crépeau and L. Salvail, in: Advances in Cryptology: Proc. of Eurocrypt '95, (Springer-Verlag, 1995), p. 133.

[2] C. H. Bennett, G. Brassard, C. Crépeau, and M.-H. Skubiszewska, in Advances in Cryptology: Proc. of Crypto '91, Vol. 576 of Lecture Notes in Comp. Science, (Springer-Verlag, 1992), p. 351.

[3] C. Crépeau, J. Mod. Opt. 41, 2445 (1994).

[4] D. Mayers, Unconditionally secure quantum bit commitment is impossible, available at http://xxx.lanl.gov/abs/quant-ph/9605044.

[5] D. Mayers, The Trouble with Quantum Bit Commitment, available at http://xxx.lanl.gov/abs/quant-ph/9603015.

[6] H.-K. Lo and H.F. Chau, Is Quantum Bit Commitment Really Possible?, available at http://xxx.lanl.gov/abs/quant-ph/9603004.

[7] C.H. Bennett and G. Brassard, in: Proceedings of the IEEE International Conference on Computers, Systems, and Signal Processing, Bangalore, India (IEEE, New York, 1984), p. 175.

[8] E. Biham, M. Boyer, G. Brassard, J. van de Graaf, and T. Mor, Security of Quantum Key Distribution Against All Collective Attacks, available at http://xxx.lanl.gov/abs/quant-ph/9801022.

[9] D. Mayers and A. Yao, Unconditional security in Quantum Cryptography, available at http://xxx.lanl.gov/abs/quant-ph/9802025.

[10] C.H. Bennett, F. Bessette, G. Brassard, L. Salvail, and J. Smolin, J. Cryptology, 5, 3 (1992).

[11] A.K. Ekert, Phys. Rev. Lett. 67, 661 (1991).

[12] C.H. Bennett, G. Brassard, and N.D. Mermin, Phys. Rev. Lett. 68, 557 (1992).

[13] C.H. Bennett, Phys. Rev. Lett. 68, 3121 (1992).

[14] A.K. Ekert, J.G. Rarity, P.R. Tapster, and G.M. Palma, Phys. Rev. Lett. 69, 1293 (1992).

[15] C. Crépeau, in: Proc. 1st Intl. Conf. Theory and Applications of Cryptology, Pragocrypt '96, Prague (CTU Publishing, Prague,1996), p. 193.

[16] G. Brassard, in: Proc. 1st Intl. Conf. Theory and Applications of Cryptology, Pragocrypt '96, Prague (CTU Publishing, Prague,1996), p. 183.

[17] Available at http://www.IRO.UMontreal.ca/ crepeau/BiblioQC.html.

[18] D.R. Stinson: Cryptography, Theory and Practice, CRC Press, Boca Raton, 1995.

[19] T. Beth, D. Jungnickel, and H. Lenz: Design Theory, Bib. Institut, Zurich, 1985.

[20] M. Hendrych, M. Dušek, and O. Haderka, Acta Physica Slovaca, 46, 393 (1996).

[21] C. Fuchs, N. Gisin, R.B. Griffits, C.-S. Niu, and A. Peres, Phys. Rev. A 56, 1164 (1997).

[22] N. Lütkenhaus, Phys. Rev. A 54, 97 (1996).

[23] G. Brassard and L. Salvail, in Advances in Cryptology: Proc. of Crypto '93, Vol. 765 of Lecture Notes in Comp. Science, (Springer-Verlag, 1994), p. 410.

[24] C.H. Bennett, G. Brassard, C. Crépeau, and U.M. Maurer, IEEE Trans. Inf. Theo. 41, 1915 (1995).

[25] N. Lütkenhaus, Estimates for Practical Quantum Cryptography, Helsinki Institute of Physics Preprint Series: HIP-1998-37/TH. 
[26] N. Lütkenhaus, Dim coherent states as signal states in the BB84 protocol: Is it secure?, poster at QCM '98, Evanston, USA, August 1998.

[27] J.I. Cirac, N. Gisin, Phys. Lett. A 229, 1 (1997).

[28] D.S. Mitrinović: Analytic Inequalities, Springer-Verlag, Berlin, 1970. 